Ambiente \& Água - An Interdisciplinary Journal of Applied Science
ISSN 1980-993X - doi:10.4136/1980-993X
www.ambi-agua.net
E-mail: ambi.agua@gmail.com

\title{
Crescimento de plantas de milho em solo acrescido de vermicompostos de lodo de curtume e irrigado com água residuária de esgoto doméstico
}

\author{
doi:10.4136/ambi-agua.1625
}

Received: 11 Mar. 2015; Accepted: 01 Sep. 2015

\author{
Guilherme Malafaia $^{1^{*}}$; Aline Sueli de Lima Rodrigues ${ }^{1}$; \\ Fernando Godinho de Araújoº ${ }^{2}$ Wilson Mozena Leandro ${ }^{3}$ \\ ${ }^{1}$ Instituto Federal Goiano (IF Goiano), Urutaí, GO, Brasil \\ Departamento de Ciências Biológicas \\ ${ }^{2}$ Instituto Federal Goiano (IF Goiano), Urutaí, GO, Brasil \\ Departamento de Ciências Agrárias \\ ${ }^{3}$ Universidade Federal de Goiás (UFG), Goiânia, GO, Brasil \\ Departamento de Agricultura \\ *Autor correspondente: e-mail: guilhermeifgoiano@gmail.com, \\ alineifgoiano@gmail.com, fernando.godinho@ifgoiano.edu.br, \\ wilsonufg@gmail.com
}

\section{RESUMO}

No presente estudo objetivou-se avaliar os parâmetros de crescimento de plantas de milho (Zea mays L.) cultivadas em vaso contendo solo acrescido de vermicompostos de lodo de curtume e irrigado com água residuária de origem doméstica. $\mathrm{O}$ arranjo dos tratamentos consistiu de um fatorial 2x6 (dois tipos de irrigação e seis tratamentos de adubação), em delineamento inteiramente casualizado (DIC), com cinco repetições. Foram avaliados, quinzenalmente, a altura das plantas e o diâmetro do colmo. Os resultados indicaram que tanto a água residuária, quanto os vermicompostos de lodo de curtume são uma boa fonte de nutrientes para as plantas, favorecendo o seu crescimento. Conclui-se, portanto, que a água residuária de origem doméstica e vermicompostos de lodo de curtume podem ser considerados resíduos de interesse agrícola para a cultura do milho.

Palavras-chave: agricultura sustentável, aproveitamento de resíduos, resíduos industriais e urbanos, reuso.

\section{Growth of corn plants in soil containing tannery sludge vermicompost and irrigated with sewage wastewater}

\begin{abstract}
This study evaluated the parameters of the growth of corn plants (Zea mays L.) grown in pots containing soil plus tannery sludge vermicompost and irrigated with household wastewater. The arrangement of treatments consisted of a factorial $2 \times 6$ (two types of irrigation and six fertilization treatments) in a completely randomized design (CRD) with five repetitions. The height of the plants and the diameter of the stems were evaluated bi-weekly. The results indicated that both the wastewater and the tannery sludge vermicompost are good sources of nutrients for the plants, promoting their growth. We conclude, therefore, that
\end{abstract}


wastewater from households and tannery sludge vermicompost can be considered an agricultural waste of interest for the corn crop.

Keywords: agro-industrial wastes, recovery of waste, reuse, sustainable agriculture.

\section{INTRODUÇÃO}

Atualmente tem sido cada vez mais estimulado o desenvolvimento de pesquisas que visem, além do tratamento, também o aproveitamento dos resíduos produzidos pelas atividades agroindustriais. As questões ambientais, em especial, têm suscitado reflexões e preocupações, uma vez que os resíduos gerados têm potencial para causar danos ambientais, se não forem devidamente tratados ou destinados (Kraemer, 2014).

Nesse sentido, com o intuito de solucionar ou minimizar essa questão, o aproveitamento desses resíduos tem emergido como alternativa interessante e ambientalmente sustentável, já que pode ocorrer a diminuição da problemática ambiental que representa o descarte desses materiais no ambiente (Nunes et al., 2009).

Uma atividade geradora de resíduo potencialmente tóxico, muito comum no Brasil, refere-se ao processamento do couro bovino, por meio das indústrias curtumeiras (Godecke et al., 2012). Esses empreendimentos trazem vários benefícios em termos de geração de emprego e renda, contudo, se deparam com problemas de cunho ambiental, uma vez que o processamento do couro faz uso de diversos insumos químicos potencialmente poluidores (Luersen et al., 2012).

A problemática se agrava ainda mais quando se constata que em função da grande demanda por produtos derivados da atividade curtumeira, grandes volumes de resíduos orgânicos também são gerados (Pacheco, 2005; 2009; Pinheiro, 2005; Ganem, 2007; Godecke et al., 2012).

Nesse sentido, o uso agronômico desses materiais tem sido considerado uma opção de aproveitamento, destacando-se que boa parte deles é constituída de material orgânico eficaz na fertilização e neutralização de solos ácidos (Godecke et al., 2012). No entanto, a aplicação desses resíduos, de forma in natura, diretamente no solo, tem causado controvérsias e resultados muito divergentes em diferentes culturas agrícolas.

Dessa forma, a vermicompostagem dos resíduos de curtume surge como opção para o aproveitamento dos mesmos no meio agrícola. Conforme discutido por Vig et al. (2011), esse processo vem sendo considerado uma opção potencial na hierarquia da gestão integrada de resíduos sólidos, principalmente porque os resíduos sólidos não aproveitados podem ser transformados em compostos orgânicos nobres.

Segundo Aquino et al. (1992), o uso de vermicompostos, em determinadas culturas, pode ser mais interessante do que o uso de resíduos in natura, uma vez que a vermicompostagem pode gerar material com alto potencial agrícola.

Por outro lado, há também grandes demandas para o uso das águas residuárias, oriundas dos esgotos domésticos, produzidas diariamente. Com o surgimento de conflitos pelo uso da água e pelo fato do consumo de água para irrigação ser expressivo - no Brasil equivale a $63 \%$ do volume total (ANA, 2002) - o interesse pelo uso de esgotos sanitários em substituição ou de forma complementar às fontes normalmente usadas para irrigação, tem aumentado.

Diferentes estudos têm apontado para o potencial uso dessas águas na agricultura, devido ao fato delas aportarem nutrientes que trazem benefícios ao desenvolvimento das plantas (Fonseca et al., 2005a; 2005b, Leal et al, 2011; Fonseca et al., 2011; Andrade-Filho et al., 2013; Bonini et al., 2014; Silva et al., 2014; Monteiro et al., 2014). Para Hespanhol (2003), as águas residuárias, oriundas de esgotos domésticos, contêm nutrientes, cujos teores atendem, se não toda, pelo menos boa parte das necessidades nutricionais das plantas em geral. 
Nesse sentido, o presente estudo objetivou analisar o crescimento e desenvolvimento (por meio de medidas de altura e diâmetro caulinar) de plantas de milho (Zea mays L.) cultivadas em solo contendo vermicomposto de lodo de curtume e irrigado com água residuária de origem doméstica.

\section{MATERIAL E MÉTODOS}

O estudo foi conduzido em ambiente protegido, localizado na área experimental do Instituto Federal Goiano (IF Goiano) - Câmpus Urutaí (GO, Brasil). O solo utilizado no experimento foi retirado da camada superficial $(0-20 \mathrm{~cm})$ de uma área localizada próxima ao ambiente protegido, tendo sido classificado como Latossolo Vermelho Distrófico Típico (Tabela 1).

Os vermicompostos utilizados neste estudo resultaram da vermicompostagem de substratos constituídos de $20 \%$ lodo de curtume dos tipos caleiro e primário e $80 \%$ de esterco bovino.

Destaca-se que o lodo de caleiro utilizado refere-se aos resíduos produzidos na etapa de depilação da pele, e o lodo primário àquele proveniente da estação de tratamento primário da indústria curtumeira. A empresa concedente dos lodos trata os efluentes gerados, na etapa de curtimento do couro bovino, separadamente dos demais resíduos e efluentes produzidos. Portanto, os lodos de curtume utilizados neste trabalho não continham o elemento cromo A Tabela 1 apresenta a caracterização desses compostos, realizada de acordo com Tedesco et al. (1995).

O arranjo dos tratamentos consistiu de um fatorial $2 \times 6$ (dois tipos de irrigação e seis tratamentos de adubação), em delineamento inteiramente casualizado (DIC), com cinco repetições, totalizando sessenta unidades experimentais.

Foram estabelecidas as seguintes unidades experimentais, irrigadas com água de abastecimento (A) e com água residuária de origem doméstica (R): (T1) Solo - testemunha, sem adubação química e sem vermicompostos; (T2) Solo + NPK (T2); (T3) Solo + vermicomposto de lodo de curtume do tipo primário a 20\% (VLp20); (T4) Solo + vermicomposto de lodo de curtume do tipo primário a 20\% (VLp20) + P; (T5) Solo + vermicomposto de lodo de curtume do tipo caleiro a $20 \%$ (VLc20) e (T6) Solo + vermicomposto de lodo de curtume do tipo caleiro a $20 \%($ VLc20) + P.

A dose de NPK utilizada nos tratamentos T2 (A) e T2 (R) foi calculada com base nas necessidades nutricionais da cultura, nas concentrações de nutrientes presentes no solo e na expectativa de rendimento da cultura de $10 \mathrm{Mg} \cdot \mathrm{ha}^{-1}$, segundo Sousa e Lobato (2004). As fontes de NPK foram ureia $\left(\mathrm{CH}_{4} \mathrm{~N}_{2} \mathrm{O}\right)$, superfosfato simples $\left(\mathrm{P}_{2} \mathrm{O}_{5}\right)$ e cloreto de potássio $\left(\mathrm{K}_{2} \mathrm{O}\right)$, respectivamente.

As doses de vermicompostos de lodo de curtume acrescidas ao solo de cultivo foram calculadas com base na concentração do $\mathrm{K}$, elemento de alta concentração nos vermicompostos usados (Tabela 1) e no fornecimento de $50 \mathrm{~kg} \cdot \mathrm{ha}^{-1}$ de $\mathrm{K}_{2} \mathrm{O}$ na base.

A quantidade de $60 \mathrm{~kg} \cdot \mathrm{ha}^{-1}$ de $\mathrm{K}_{2} \mathrm{O}$ foi fornecida via adubação de cobertura, em duas parcelas de $30 \mathrm{~kg} \cdot \mathrm{ha}^{-1}$, aos 40 e 60 dias após a semeadura (DAS). Assim, a dose do vermicomposto de lodo de caleiro (VLc20) acrescida ao solo de cultivo correspondeu a 6,1 Mg.ha ${ }^{-1}$, e do vermicomposto de lodo primário (VLp20), 5,5 Mg.ha ${ }^{-1}$. Não foi necessária a realização da correção do $\mathrm{pH}$ do solo de cultivo.

O solo, previamente misturado com os vermicompostos (VLc20 e VLp20) e insumos, foi acondicionado em vasos de polietileno (capacidade volumétrica de $15 \mathrm{~L}$ ) em um total de 12,5 kg. Logo após a instalação das unidades experimentais, os vasos foram semeados com três sementes de milho (Zea mays L.), cultivar LG 6036 (LG Semente ${ }^{\circledR}$ ) e, 15 dias depois, realizou-se o desbaste, mantendo-se uma planta por vaso. 
Os tratos fitossanitários foram realizados quando necessários e a adubação nitrogenada de cobertura (total de $130 \mathrm{~kg} \cdot \mathrm{ha}^{-1}$ ) foi realizada superficialmente, em duas parcelas iguais $\left(65 \mathrm{~kg} \cdot \mathrm{ha}^{-1}\right)$, aos 40 e 60 DAS.

Tabela 1. Principais características iniciais do solo (Latossolo Vermelho Distrófico Típico) e dos vermicompostos de lodo de curtume utilizados como fontes de nutrientes para o desenvolvimento de plantas de milho (Zea mays). Urutaí, GO, 2014.

\begin{tabular}{|c|c|c|c|}
\hline \multirow[b]{2}{*}{ Variáveis } & \multicolumn{3}{|c|}{ Resultados } \\
\hline & Solo & $\begin{array}{l}\text { Vermicomposto } \\
\text { (Lc20)* }\end{array}$ & $\begin{array}{l}\text { Vermicomposto } \\
\text { (Lp20)* }\end{array}$ \\
\hline $\mathrm{pH}\left(\mathrm{CaCl}_{2}\right)$ & 5,30 & 8,8 & 8,8 \\
\hline $\mathrm{N}(\%)$ & 0,11 & 1,5 & 1,2 \\
\hline $\mathrm{P}\left(\right.$ Melich $\left.-\mathrm{mg} \cdot \mathrm{dm}^{-3}\right)$ & 5,00 & 700,0 & 400,0 \\
\hline $\mathrm{K}\left(\mathrm{mg} \cdot \mathrm{dm}^{-3}\right)$ & 240,00 & $18.000,0$ & $20.000,0$ \\
\hline $\mathrm{Ca}\left(\mathrm{cmolc} \cdot \mathrm{dm}^{-3}\right)$ & 2,60 & 14,0 & 14,0 \\
\hline $\operatorname{Mg}\left(\right.$ cmolc. $\left.\mathrm{dm}^{-3}\right)$ & 0,80 & 18,0 & 15,0 \\
\hline $\mathrm{Ca}+\mathrm{Mg}\left(\mathrm{cmolc} \cdot \mathrm{dm}^{-3}\right)$ & 3,40 & 32,0 & 29,0 \\
\hline $\mathrm{Al}\left(\mathrm{cmolc} \mathrm{dm}^{-3}\right)$ & 0,00 & 0,0 & 0,0 \\
\hline $\mathrm{H}+\mathrm{Al}\left(\mathrm{cmolc} \cdot \mathrm{dm}^{-3}\right)$ & 2,20 & 0,0 & 0,0 \\
\hline CTC (cmolc. $\left.\mathrm{dm}^{-3}\right)$ & 6,20 & 82,4 & 85,4 \\
\hline $\mathrm{Na}\left(\mathrm{mg} \cdot \mathrm{dm}^{-3}\right)$ & 8,00 & 0,0 & $1.200,0$ \\
\hline $\mathrm{Cu}\left(\mathrm{mg} \cdot \mathrm{dm}^{-3}\right)$ & 2,50 & 5,0 & 2,9 \\
\hline $\mathrm{Fe}\left(\mathrm{mg} \cdot \mathrm{dm}^{-3}\right)$ & 63,00 & 244,0 & 122,0 \\
\hline $\operatorname{Mn}\left(\mathrm{mg} \cdot \mathrm{dm}^{-3}\right)$ & 47,00 & 68,0 & 55,0 \\
\hline $\mathrm{Zn}\left(\mathrm{mg} \cdot \mathrm{dm}^{-3}\right)$ & 4,40 & 36,0 & 39,0 \\
\hline Matéria orgânica (\%) & 2,30 & 29,9 & 24,2 \\
\hline Sat $\mathrm{Al}(\%)$ & 0,00 & 0,0 & 0,0 \\
\hline Sat Base (\%) & 65,00 & 100,0 & 100,0 \\
\hline $\mathrm{Ca} / \mathrm{Mg}(\%)$ & 03,30 & 0,8 & 0,9 \\
\hline $\mathrm{Ca} / \mathrm{CTC}(\%)$ & 42,00 & 17,0 & 16,0 \\
\hline $\mathrm{Mg} / \mathrm{CTC}(\%)$ & 13,00 & 22,0 & 18,0 \\
\hline K/CTC (\%) & 10,00 & 56,0 & 60,0 \\
\hline $\mathrm{H}+\mathrm{Al} / \mathrm{CTC}(\%)$ & 35,00 & 0,0 & 0,0 \\
\hline Argila (\%) & 27,00 & - & - \\
\hline Silte $(\%)$ & 15,00 & - & - \\
\hline Areia $(\%)$ & 58,00 & - & - \\
\hline Condutividade Elétrica $\left(\mu \mathrm{S} \mathrm{cm}^{-3}\right)$ & 184,00 & $1.170,0$ & $1.850,0$ \\
\hline Carbono Orgânico Total (\%) & 1,30 & 17,3 & 14,0 \\
\hline Densidade de partícula $\left(\mathrm{g} \cdot \mathrm{cm}^{-3}\right)$ & 2,45 & - & - \\
\hline $\mathrm{Cr}\left(\mathrm{mg} \cdot \mathrm{dm}^{-3}\right)$ & $<5,00$ & $<5,0$ & $<5,0$ \\
\hline
\end{tabular}

*Vermicomposto (Lc20): vermicomposto de lodo de curtume constituído de $20 \%$ de lodo de curtume do tipo caleiro e $80 \%$ de esterco bovino. Vermicomposto (Lp20): vermicomposto de lodo de curtume constituído de $20 \%$ de lodo de curtume do tipo primário e $80 \%$ de esterco bovino. 
A água utilizada nas irrigações era proveniente do sistema de abastecimento de água do IF Goiano - Câmpus Urutaí, tratada em uma Estação de Tratamento de Água (ETA) própria do câmpus, e água residuária oriunda de uma lagoa de estabilização de esgoto doméstico, também localizada nas dependências da instituição. Para a caracterização das águas de irrigação foram coletadas amostras mensais, ao longo do período experimental $(\mathrm{n}=4)$, para avaliação de parâmetros físicos, químicos e físico-químicos, conforme metodologia proposta pela APHA et al. (1997) (Tabela 2).

Tabela 2. Caracterização física, química e físico-química das águas de abastecimento e residuária utilizadas na irrigação de plantas de milho (Zea mays), cultivar LG 6036. Urutaí-GO, 2014

\begin{tabular}{|c|c|c|}
\hline Parâmetros & $\begin{array}{c}\text { Água de } \\
\text { abastecimento* }\end{array}$ & Água residuária* \\
\hline $\mathrm{pH}$ a $25^{\circ} \mathrm{C}$ & 7,38 & 7,73 \\
\hline Turbidez (UNT) & 2,85 & 601,67 \\
\hline Fe dissolvido (mg. $\left.\mathrm{L}^{-1}\right)$ & 0,31 & 1,26 \\
\hline $\mathrm{N}$ total $\left(\mathrm{mg} \cdot \mathrm{L}^{-1}\right)$ & 2,43 & 54,57 \\
\hline $\mathrm{N}$ orgânico & ND & 4,90 \\
\hline $\mathrm{N}$ amoniacal $\left(\mathrm{mg} \cdot \mathrm{L}^{-1}\right)$ & 0,07 & 43,67 \\
\hline Nitrato $\left(\mathrm{mg} \cdot \mathrm{L}^{-1}\right)$ & 0,33 & 6,00 \\
\hline Condutividade elétrica a $25^{\circ} \mathrm{C}\left(\mu \mathrm{s} . \mathrm{cm}^{-1}\right)$ & 67,20 & 746,33 \\
\hline$P$ total $\left(\mathrm{mg} . \mathrm{L}^{-1}\right)$ & 0,14 & 9,10 \\
\hline Ortofosfato (mg. $\left.\mathrm{L}^{-1}\right)$ & 0,33 & 20,86 \\
\hline DBO (mg.L $\left.\mathrm{L}^{-1}\right)$ & 0,67 & 572,11 \\
\hline Sólidos totais $\left(\mathrm{mg} \cdot \mathrm{L}^{-1}\right)$ & 73,33 & $1.290,00$ \\
\hline $\mathrm{Cu}$ dissolvido (mg. $\left.\mathrm{L}^{-1}\right)$ & 0,35 & 0,44 \\
\hline $\mathrm{Zn}\left(\mathrm{mg} \cdot \mathrm{L}^{-1}\right)$ & 0,37 & 0,26 \\
\hline $\mathrm{Na}\left(\mathrm{mg} \cdot \mathrm{L}^{-1}\right)$ & 25,59 & 56,63 \\
\hline Mn dissolvido (mg. $\mathrm{L}^{-1}$ ) & 0,57 & 1,80 \\
\hline Mg dissolvido (mg. $\left.\mathrm{L}^{-1}\right)$ & 1,78 & 12,16 \\
\hline $\mathrm{Ca}\left(\mathrm{mg} \cdot \mathrm{L}^{-1}\right)$ & 6,28 & 37,41 \\
\hline $\mathrm{S}\left(\mathrm{mg} \cdot \mathrm{L}^{-1}\right)$ & 3,00 & 3,10 \\
\hline $\mathrm{K}\left(\mathrm{mg} \cdot \mathrm{L}^{-1}\right)$ & 7,45 & 19,16 \\
\hline COT $\left(\mathrm{mg} . \mathrm{L}^{-1}\right)$ & 14,95 & 43,47 \\
\hline
\end{tabular}

Legenda: ND: parâmetro não dosado.

* Os valores apresentados referem-se à média de quatro amostras coletadas ao longo do período experimental.

O manejo da irrigação da cultura foi realizado a partir de um tanque evaporímetro desenvolvido por Salomão (2012), de forma circular, diâmetro interno de $52 \mathrm{~cm}$ e altura (interna) de $24 \mathrm{~cm}$, instalado sob um estrado de madeira de $15 \mathrm{~cm}$ de altura e colocado no interior do ambiente protegido, entre os tratamentos.

O volume de água irrigado diariamente, para manter a capacidade de retenção de água do solo em $70 \%\left(243,1 \mathrm{~mL} \cdot \mathrm{kg}^{-1}\right)$, durante o experimento, baseou-se na área do vaso a ser irrigado $\left(0,06 \mathrm{~m}^{2}\right)$ e na evapotranspiração da cultura (ETc). 
Destaca-se que o volume de água a ser reposto era medido em uma proveta graduada. A capacidade de retenção de água do solo $\left(\mathrm{C}_{100 \%}=347,4 \mathrm{~mL} \cdot \mathrm{kg}^{-1}\right)$ foi determinada por meio do cálculo do poder de embebição do solo, conforme metodologia preconizada pela Embrapa (1997).

Quanto às determinações, avaliou-se, quinzenalmente, ao longo de 120 dias do experimento, a altura da planta (medida da superfície do solo até a base da última folha) e diâmetro do colmo (medido a $1 \mathrm{~cm}$ de altura em relação à superfície do solo), utilizando-se régua milimetrada e paquímetro digital, respectivamente.

Os dados foram submetidos à análise de variância e, quando significativos, foram submetidos à análise de regressão e, posteriormente, elaborados os gráficos com os modelos de regressão que melhor se ajustariam aos dados amostrais. Nesses casos, a seleção do melhor modelo de regressão foi baseada no coeficiente de determinação $\left(R^{2}\right)$ e a correspondente interpretação do modelo.

A normalidade residual foi verificada pelo teste de Shapiro-Wilk e o teste de Bartlett foi utilizado para verificar a homocedasticidade residual. A análise de variância foi realizada por meio do software ASSISTAT, versão 7.7 beta (cópia distribuída gratuitamente). As representações gráficas dos modelos de regressão escolhidos foram confeccionadas no software R versão 3.0.3 (R Core Team, 2014).

\section{RESULTADOS E DISCUSSÃO}

Em relação às variáveis avaliadas quinzenalmente, observou-se interação entre as fontes de variação "irrigação" e "tratamentos" em todas as medidas de altura das plantas (m) realizadas ao longo do período experimental (aos 15, 30, 45, 60, 75, 90, 105 e 120 DAS), com exceção apenas para a medida realizada aos 30 DAS (Tabela 3). O desdobramento da interação tipo de água de "irrigação" x "tratamentos de adubação" para a variável altura (m) do milho pode ser observado na Tabela 4.

Aos 15 DAS, notou-se que os tratamentos T1R (testemunha), T2R (adubação química) e T3R (solo + VLp20), irrigados com água residuária, apresentaram maior altura, em relação à altura das plantas dos demais tratamentos. As plantas dos tratamentos T6A e T6R (solo + VLc20 + P), irrigadas ou não com água residuária, nessa mesma época, apresentaram maior altura, quando comparadas com a altura das plantas dos demais tratamentos (Tabela 4).

Tabela 3. Resumo do teste $\mathrm{F}$ da variância para a variável altura $(\mathrm{m})$ das plantas de milho (Zea mays - LG 6036), em função do tipo de água de irrigação e dos tratamentos de adubação. Urutaí, GO, 2014.

\begin{tabular}{lcccccccc}
\hline \multirow{2}{*}{ Fatores } & \multicolumn{8}{c}{ Período experimental (DAS) } \\
\cline { 2 - 9 } & 15 & 30 & 45 & 60 & 75 & 90 & 105 & 120 \\
\hline $\begin{array}{l}\text { Fator 1 (tipos de } \\
\text { irrigação) }\end{array}$ & $28,50^{* *}$ & $93,90^{* *}$ & $02,85^{\text {ns }}$ & $114,81^{* *}$ & $349,05^{* *}$ & $87,96^{* *}$ & $37,87^{* *}$ & $29,95^{* *}$ \\
$\begin{array}{l}\text { Fator 2 } \\
\text { (tratamentos) }\end{array}$ & $21,78^{* *}$ & $11,76^{* *}$ & $18,56^{* *}$ & $94,75^{* *}$ & $185,42^{* *}$ & $44,25^{* *}$ & $18,97^{* *}$ & $14,82^{* *}$ \\
$\begin{array}{l}\text { Interação (fator 1 x } \\
\text { fator 2) }\end{array}$ & $2,93^{*}$ & $2,10^{\text {ns }}$ & $7,64^{* *}$ & $26,55^{* *}$ & $136,09^{* *}$ & $32,15^{* *}$ & $12,96^{* *}$ & $10,00^{* *}$ \\
CV (\%) & 16,65 & 13,03 & 11,87 & 5,62 & 3,12 & 5,58 & 7,98 & 8,80 \\
\hline
\end{tabular}

Legenda: *: significativo a $5 \%$ de probabilidade; **: significativo a $1 \%$ de probabilidade; ns: não significativo; CV: coeficiente de variação, expresso em percentual. Tipos de irrigação: água de abastecimento e água residuária de origem doméstica. Tratamentos: Solo - testemunha (sem adubação química e sem vermicompostos); Solo + NPK; Solo + vermicomposto de lodo de lodo de curtume do tipo primário a 20\% (VLp20); Solo + vermicomposto de lodo de curtume do tipo primário a 20\% (VLp20) + P; Solo + vermicomposto de lodo de lodo de curtume do tipo caleiro a $20 \%$ (VLc20); Solo + vermicomposto de lodo de curtume do tipo caleiro a $20 \%$ $(\mathrm{VLc} 20)+\mathrm{P}$. 
Tabela 4. Valores médios do desdobramento da interação tipo de água de irrigação x tratamentos de adubação para a variável altura (cm) das plantas de milho (Zea mays L. - LG 6036). Urutaí, GO, 2014.

\begin{tabular}{|c|c|c|c|c|c|c|}
\hline \multirow{2}{*}{$\begin{array}{l}\text { Tipos de } \\
\text { irrigaçãao }\end{array}$} & \multicolumn{6}{|c|}{ Tratamentos } \\
\hline & $\begin{array}{c}\text { Solo } \\
\text { (testemunha) }\end{array}$ & $\begin{array}{l}\text { Solo + } \\
\text { NPK }\end{array}$ & $\begin{array}{l}\text { Solo + } \\
\text { VLp20 }\end{array}$ & $\begin{array}{c}\text { Solo + } \\
\text { VLp20 + P }\end{array}$ & $\begin{array}{l}\text { Solo + } \\
\text { VLc20 }\end{array}$ & $\begin{array}{c}\text { Solo + } \\
\text { VLc20 + P }\end{array}$ \\
\hline \multicolumn{7}{|c|}{15 dias } \\
\hline Abastecimento & $0,24 \mathrm{bC}$ & $0,30 \mathrm{bBC}$ & $0,30 \mathrm{bBC}$ & $0,38 \mathrm{aB}$ & $0,32 \mathrm{aBC}$ & $0,62 \mathrm{aA}$ \\
\hline Residuária & $0,40 \mathrm{aB}$ & $0,44 \mathrm{aB}$ & $0,44 \mathrm{aB}$ & $0,46 \mathrm{aAB}$ & $0,40 \mathrm{aB}$ & $0,58 \mathrm{aA}$ \\
\hline \multicolumn{7}{|c|}{45 dias } \\
\hline Abastecimento & $0,51 \mathrm{bB}$ & $1,14 \mathrm{aA}$ & $1,24 \mathrm{aA}$ & $1,12 \mathrm{aA}$ & $1,10 \mathrm{aA}$ & $1,24 \mathrm{aA}$ \\
\hline Residuária & $1,01 \mathrm{aAB}$ & $1,18 \mathrm{aAB}$ & $1,20 \mathrm{aAB}$ & $1,11 \mathrm{aAB}$ & $0,96 \mathrm{aB}$ & $1,22 \mathrm{aA}$ \\
\hline \multicolumn{7}{|c|}{60 dias } \\
\hline Abastecimento & $0,58 \mathrm{bD}$ & $1,36 \mathrm{bBC}$ & $1,32 \mathrm{bC}$ & $1,55 \mathrm{aA}$ & $1,47 \mathrm{bAB}$ & $1,58 \mathrm{aA}$ \\
\hline Residuária & $1,30 \mathrm{aB}$ & $1,54 \mathrm{aA}$ & $1,58 \mathrm{aA}$ & $1,53 \mathrm{aA}$ & $1,58 \mathrm{aA}$ & $1,68 \mathrm{aA}$ \\
\hline \multicolumn{7}{|c|}{75 dias } \\
\hline Abastecimento & $0,63 \mathrm{bD}$ & $1,38 \mathrm{bC}$ & $1,48 \mathrm{bB}$ & $1,54 \mathrm{aAB}$ & $1,53 \mathrm{aAB}$ & $1,58 \mathrm{bA}$ \\
\hline Residuária & $1,53 \mathrm{aB}$ & $1,54 \mathrm{aB}$ & $1,58 \mathrm{aB}$ & $1,56 \mathrm{aB}$ & $1,56 \mathrm{aB}$ & $1,68 \mathrm{aA}$ \\
\hline \multicolumn{7}{|c|}{90 dias } \\
\hline Abastecimento & $0,74 \mathrm{bC}$ & $1,38 \mathrm{bB}$ & $1,48 \mathrm{aAB}$ & $1,54 \mathrm{aA}$ & $1,53 \mathrm{aAB}$ & $1,58 \mathrm{aA}$ \\
\hline Residuária & $1,53 \mathrm{aA}$ & $1,54 \mathrm{aA}$ & $1,57 \mathrm{aA}$ & $1,56 \mathrm{aA}$ & $1,56 \mathrm{aA}$ & $1,68 \mathrm{aA}$ \\
\hline \multicolumn{7}{|c|}{105 dias } \\
\hline Abastecimento & $0,80 \mathrm{bB}$ & $1,38 \mathrm{bA}$ & $1,48 \mathrm{aA}$ & $1,54 \mathrm{aA}$ & $1,53 \mathrm{aA}$ & $1,58 \mathrm{aA}$ \\
\hline Residuária & $1,53 \mathrm{aA}$ & $1,54 \mathrm{aA}$ & $1,57 \mathrm{aA}$ & $1,56 \mathrm{aA}$ & $1,56 \mathrm{aA}$ & $1,68 \mathrm{aA}$ \\
\hline \multicolumn{7}{|c|}{120 dias } \\
\hline Abastecimento & $0,82 \mathrm{bB}$ & $1,38 \mathrm{aA}$ & $1,48 \mathrm{aA}$ & $1,54 \mathrm{aA}$ & $1,53 \mathrm{aA}$ & $1,58 \mathrm{aA}$ \\
\hline Residuária & $1,53 \mathrm{aA}$ & $1,54 \mathrm{aA}$ & $1,57 \mathrm{aA}$ & $1,56 \mathrm{aA}$ & $1,56 \mathrm{aA}$ & $1,68 \mathrm{aA}$ \\
\hline
\end{tabular}

Nota: Médias seguidas pela mesma letra minúscula na coluna e maiúscula na linha não diferem entre si pelo teste de Tukey a $5 \%$ de probabilidade.

A partir do $90^{\circ}$ dia experimental, os tratamentos $\mathrm{T} 3$ (solo + LLp20), T4 $($ solo + VLp20 + P), T5 (solo + VLc20) e T6 (solo + VLc20 + P) não diferiram entre si, quando irrigados ou não com água residuária (Tabela 4).

Esses resultados podem estar relacionados ao fato de que o crescimento das plantas de milho, em condições adequadas, ocorre apenas na fase vegetativa. Quando a cultura entra na fase reprodutiva o crescimento se estabiliza, não havendo, portanto, acréscimo significativo na altura das plantas de milho. No entanto, há de se ressaltar que para todas as épocas de avaliação observou-se acréscimo significativo na altura das plantas do tratamento testemunha (T1R), irrigadas com água residuária, quando comparadas às do tratamento testemunha (T1A), irrigado com água de abastecimento.

A análise de regressão revelou significância e altos valores de $\mathrm{R}^{2}$ para os diferentes modelos em relação à altura das plantas ao longo do período experimental (Tabela 5). No entanto, o modelo que melhor se ajustou à maioria dos dados de altura das plantas foi o de regressão quadrática de resposta platô (Figura 1), com valores de $\mathrm{R}^{2}$ iguais ou superiores a 
97\%. Este modelo é representado por uma fase crescente, descrita por uma equação do segundo grau e, após a estabilização, por um platô.

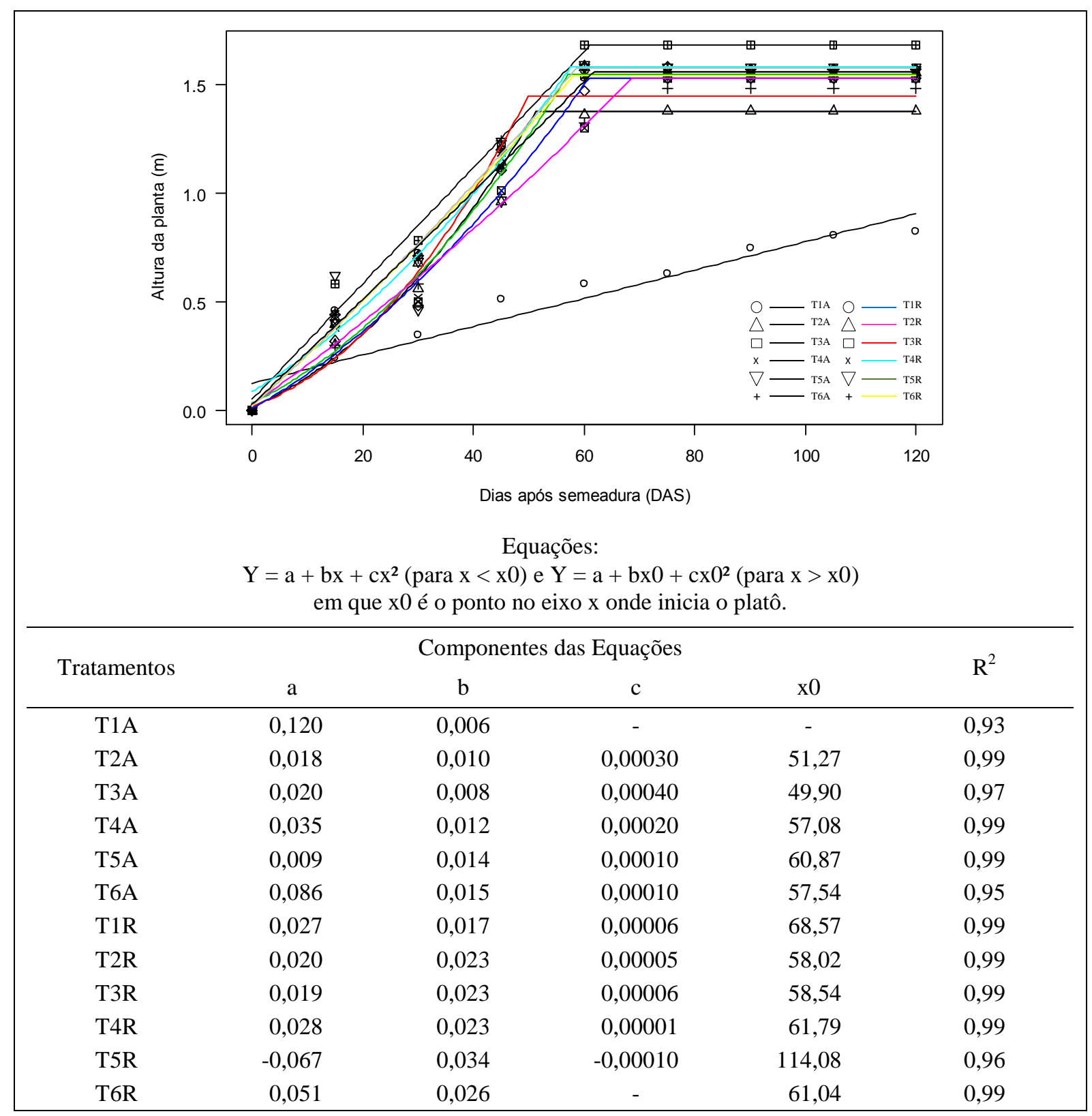

Figura 1. Representação gráfica e equações dos modelos de regressão que melhor se ajustaram aos resultados de altura das plantas (m) de milho (Zea mays) (LG 6036), obtidos ao longo do período experimental. Urutaí, GO, 2014. Legenda: $R^{2}$ : Coeficientes de determinação.

O tratamento testemunha (T1A), irrigado com água de abastecimento e o tratamento T6R (irrigado com água residuária) constituem as exceções (Figura 1). No primeiro, o modelo de regressão que melhor se ajustou aos dados foi o de regressão linear simples, com $\mathrm{R}^{2}$ de $93 \%$ (Figura 1). Ressalta-se que esse resultado pode estar relacionado ao fato de que apenas uma planta do tratamento T1A (testemunha irrigado com água de abastecimento) atingiu ao estágio reprodutivo, apresentando inflorescência. As demais plantas não completaram seu ciclo vegetativo e, possivelmente, isso explica o crescimento linear da cultura do referido tratamento ao longo do período experimental.

No entanto, o tratamento T6R (irrigado com água residuária) apresentou um modelo de regressão linear de resposta platô, o qual possui dois segmentos, dos quais o primeiro descreve 
uma reta crescente, até o $61^{\circ}$ dia experimental, considerado o início do platô. A partir desse ponto, o valor da altura da planta é constante, caracterizando o segundo segmento (Figura 1).

Tabela 5. Resumo da análise de regressão (valores de $\mathrm{R}^{2}$ ) para a variável altura das planas de milho (Zea mays - LG 6036), em função dos dias após a germinação (15, 30, 45, 60, 75, 90, 105 e 120 dias). Urutaí, GO, 2014.

\begin{tabular}{lcccccc}
\hline \multirow{2}{*}{$\begin{array}{l}\text { Modelos de } \\
\text { regressão }\end{array}$} & \multicolumn{6}{c}{ Irrigação com água de abastecimento (A) } \\
\cline { 2 - 7 } & $\begin{array}{c}\text { Solo } \\
\text { (testemunha) }\end{array}$ & $\begin{array}{c}\text { Solo }+ \\
\text { NPK }\end{array}$ & $\begin{array}{c}\text { Solo }+ \\
\text { VLp20 }\end{array}$ & $\begin{array}{c}\text { Solo }+ \\
\text { VLp20 + }\end{array}$ & $\begin{array}{c}\text { Solo }+ \\
\text { VLc20 }\end{array}$ & $\begin{array}{r}\text { Solo + } \\
\text { VLc20 + P }\end{array}$ \\
\hline Reg. linear & $0,93^{\text {ns }}$ & 0,78 & 0,80 & 0,80 & 0,82 & 0,76 \\
Reg. quadrática & $0,99^{\text {ns }}$ & 0,96 & 0,97 & 0,95 & 0,96 & 0,92 \\
MLRP & - & - & - & - & - & - \\
MQRP & - & 0,99 & 0,97 & 0,99 & 0,99 & 0,95 \\
CV (\%) & 42,37 & 5,93 & 3,62 & 3,29 & 4,63 & 5,04 \\
\cline { 2 - 7 } & & & Irrigação com água residuária (R) & \\
Reg. linear & 0,86 & 0,79 & 0,79 & 0,81 & 0,81 & 0,79 \\
Reg. quadrática & 0,97 & 0,98 & 0,97 & 0,98 & 0,96 & 0,97 \\
MLRP & - & - & - & - & - & 0,99 \\
MQRP & 0,99 & 0,99 & 0,99 & 0,99 & 0,96 & - \\
CV (\%) & 9,51 & 2,06 & 6,34 & 7,48 & 2,86 & 4,61 \\
\hline
\end{tabular}

Legenda: (-): modelo não ajustado, ns: não significativo, Reg: regressão; MLRP: modelo de regressão linear de resposta platô, MQRP: modelo de regressão quadrática de resposta platô.

Em relação à variável diâmetro do colmo $(\mathrm{cm})$ aferida quinzenalmente, também observou-se que houve interação entre as fontes de variação "irrigação" e "tratamentos" em todas as épocas de medição realizadas (aos 15, 30, 45, 60, 75, 90, 105 e 120 DAS), com exceção apenas para a medida realizada aos 60 DAS (Tabela 6).

Tabela 6. Resumo do teste $\mathrm{F}$ da análise de variância para a variável diâmetro do colmo $(\mathrm{cm})$ das plantas de milho (Zea mays L. - LG 6036), em função do tipo de água de irrigação e dos tratamentos de adubação. Urutaí, GO, 2014.

\begin{tabular}{lcccccccc}
\hline \multirow{2}{*}{ Fatores } & \multicolumn{7}{c}{ Período experimental (dias) } \\
\cline { 2 - 9 } & 15 & 30 & 45 & 60 & 75 & 90 & 105 & 120 \\
\hline Fator 1 (tipos de irrigação) & $39,76^{*}$ & $31,94^{*}$ & $38,74^{*}$ & $11,57^{*}$ & $22,34^{*}$ & $17,70^{*}$ & $14,30^{*}$ & $10,96^{*}$ \\
Fator 2 (tratamentos) & $13,90^{*}$ & $24,56^{*}$ & $21,26^{*}$ & $13,84^{*}$ & $29,15^{*}$ & $25,37^{*}$ & $20,33^{*}$ & $20,74^{*}$ \\
Interação (fator 1 x fator 2) & $3,78^{*}$ & $2,65^{*}$ & $3,14^{*}$ & $2,25^{\text {ns }}$ & $9,03^{*}$ & $11,06^{*}$ & $10,96^{*}$ & $11,59^{*}$ \\
CV (\%) & 14,47 & 14,06 & 12,90 & 12,51 & 10,58 & 10,39 & 10,84 & 10,97 \\
\hline
\end{tabular}

Legenda: *: significativo a 5\% de probabilidade; CV $(\%)$ : coeficiente de variação, expressa em porcentagem. Tratamentos: Solo - testemunha (sem adubação química e sem vermicompostos); Solo + NPK; Solo + vermicomposto de lodo de curtume do tipo primário a 20\% (VLp20); Solo + vermicomposto de lodo de curtume do tipo primário a 20\% (VLp20) + P; Solo + vermicomposto de lodo de curtume do tipo caleiro a 20\% (VLc20); Solo + vermicomposto de lodo de curtume do tipo caleiro a 20\% (VLc20) + P. 
A precisão experimental estimada pelo $\mathrm{CV}$ apresentou bom nível, sendo que o maior valor foi observado para o diâmetro do colmo medido no $15^{\circ}$ dia experimental $(\mathrm{CV}=14,47)$. O desdobramento da interação "tipo de água de irrigação" x "tratamentos de adubação" para essa variável pode ser observado na Tabela 7.

Tabela 7. Valores médios do desdobramento da interação tipo de água de irrigação $\mathrm{x}$ tratamentos de adubação para a variável diâmetro do colmo $(\mathrm{cm})$ de plantas de milho (Zea mays L. - LG 6036). Urutaí, GO, 2014.

\begin{tabular}{|c|c|c|c|c|c|c|}
\hline \multicolumn{7}{|c|}{ Tratamentos } \\
\hline \multirow{2}{*}{$\begin{array}{l}\text { Tipos de } \\
\text { irrigação }\end{array}$} & \multicolumn{5}{|c|}{15 dias } & \multirow[b]{2}{*}{$\begin{array}{c}\text { Solo + } \\
\text { VLc20 + P }\end{array}$} \\
\hline & $\begin{array}{c}\text { Solo } \\
\text { (testemunha) }\end{array}$ & Solo + NPK & $\begin{array}{l}\text { Solo + } \\
\text { VLp20 }\end{array}$ & $\begin{array}{c}\text { Solo + } \\
\text { VLp20 + P }\end{array}$ & $\begin{array}{l}\text { Solo + } \\
\text { VLc20 }\end{array}$ & \\
\hline Abastecimento & $0,36 \mathrm{bC}$ & $0,50 \mathrm{bBC}$ & $0,44 \mathrm{bBC}$ & $0,60 \mathrm{bB}$ & $0,52 \mathrm{bBC}$ & $0,80 \mathrm{aA}$ \\
\hline \multirow[t]{2}{*}{ Residuária } & $0,58 \mathrm{aB}$ & $0,64 \mathrm{aAB}$ & $0,72 \mathrm{aAB}$ & $0,72 \mathrm{aAB}$ & $0,66 \mathrm{aAB}$ & $0,76 \mathrm{aA}$ \\
\hline & & & 30 dias & & & \\
\hline Abastecimento & $0,63 \mathrm{bC}$ & $1,26 \mathrm{aAB}$ & $1,00 \mathrm{Bb}$ & $1,54 \mathrm{aA}$ & $1,40 \mathrm{Aa}$ & $1,56 \mathrm{aA}$ \\
\hline \multirow[t]{2}{*}{ Residuária } & $1,00 \mathrm{aB}$ & $1,50 \mathrm{aA}$ & $1,64 \mathrm{aA}$ & $1,74 \mathrm{aA}$ & $1,50 \mathrm{aA}$ & $1,70 \mathrm{aA}$ \\
\hline & & & 45 dias & & & \\
\hline Abastecimento & $0,79 \mathrm{bC}$ & $1,86 \mathrm{aA}$ & $1,32 \mathrm{Bb}$ & $1,96 \mathrm{aA}$ & $1,76 \mathrm{aA}$ & $1,78 \mathrm{bA}$ \\
\hline \multirow[t]{2}{*}{ Residuária } & $1,50 \mathrm{aB}$ & $2,02 \mathrm{aA}$ & $1,94 \mathrm{aA}$ & $2,06 \mathrm{aA}$ & $1,96 \mathrm{aA}$ & $2,18 \mathrm{aA}$ \\
\hline & & & 75 dias & & & \\
\hline Abastecimento & $0,74 \mathrm{bB}$ & $2,19 \mathrm{aA}$ & $1,92 \mathrm{Aa}$ & $2,18 \mathrm{aA}$ & $1,89 \mathrm{aA}$ & $1,85 \mathrm{aA}$ \\
\hline \multirow[t]{2}{*}{ Residuária } & $1,75 \mathrm{aB}$ & $2,12 \mathrm{aAB}$ & $2,16 \mathrm{aA}$ & $2,22 \mathrm{aA}$ & $2,06 \mathrm{aAB}$ & $1,96 \mathrm{aAB}$ \\
\hline & & & 90 dias & & & \\
\hline Abastecimento & $0,80 \mathrm{bB}$ & $2,22 \mathrm{aA}$ & $1,98 \mathrm{aA}$ & $2,24 \mathrm{aA}$ & $1,89 \mathrm{aA}$ & $1,93 \mathrm{aA}$ \\
\hline \multirow[t]{2}{*}{ Residuária } & $1,86 \mathrm{aA}$ & $2,12 \mathrm{aA}$ & $2,16 \mathrm{aA}$ & $2,22 \mathrm{aA}$ & $2,06 \mathrm{aA}$ & $1,96 \mathrm{aA}$ \\
\hline & & & 105 dias & & & \\
\hline Abastecimento & $0,84 \mathrm{bB}$ & $2,20 \mathrm{aA}$ & $2,04 \mathrm{aA}$ & $2,28 \mathrm{aA}$ & $1,91 \mathrm{aA}$ & $1,99 \mathrm{aA}$ \\
\hline \multirow[t]{2}{*}{ Residuária } & $1,95 \mathrm{aA}$ & $2,14 \mathrm{aA}$ & $2,16 \mathrm{aA}$ & $2,23 \mathrm{aA}$ & $2,08 \mathrm{aA}$ & $1,96 \mathrm{aA}$ \\
\hline & & & 120 dias & & & \\
\hline Abastecimento & $0,84 \mathrm{bB}$ & $2,26 \mathrm{aA}$ & $2,10 \mathrm{aA}$ & $2,32 \mathrm{aA}$ & $1,92 \mathrm{aA}$ & $2,08 \mathrm{aA}$ \\
\hline Residuária & $1,98 \mathrm{aA}$ & $2,28 \mathrm{aA}$ & $2,18 \mathrm{aA}$ & $2,19 \mathrm{aA}$ & $2,04 \mathrm{aA}$ & $1,98 \mathrm{aA}$ \\
\hline
\end{tabular}

Nota: Médias seguidas pela mesma letra minúscula na coluna e maiúscula na linha não diferem entre si pelo teste de Tukey a $5 \%$ de probabilidade.

O tratamento testemunha (T1R) irrigado com água residuária apresentou acréscimo do diâmetro do colmo em todas as épocas de avaliações realizadas, em relação ao tratamento testemunha irrigado com água de abastecimento (Tabela 7). Quanto aos demais tratamentos irrigados com água residuária (T2R a T6R), não foi observada diferença estatística entre os mesmos, em nenhuma época de avaliação.

Nos tratamentos irrigados com água de abastecimento, observou-se maior diâmetro do colmo nas plantas do tratamento T6A aos 15 DAS (Tabela 7). Aos 30 DAS, destacaram-se os tratamentos T4A, T5A e T6A, os quais apresentaram maior diâmetro do colmo em relação às 
plantas dos tratamentos T1A e T3A e diâmetro semelhante ao das plantas que receberam adubação mineral (grupo T2A).

Aos 45 DAS, as plantas dos tratamentos T2A, T4A, T5A e T6A apresentaram maior diâmetro do colmo, em relação aos tratamentos T1A e T3A. A partir do $75^{\circ}$ dia experimental, não ocorreram diferenças significativas entre os tratamentos irrigados com água de abastecimento (Tabela 7), provavelmente devido à elongação dos entre nós das plantas e, consequentemente, estabilização do diâmetro do colmo, com exceção do tratamento T1A, que foi sempre menor.

A análise de regressão para a variável diâmetro do colmo também revelou significância e altos valores de $\mathrm{R}^{2}$ para diferentes modelos, ao longo do período experimental (Tabela 8).

Tabela 8. Resumo da análise de regressão (valores de $\mathrm{R}^{2}$ ) para a variável diâmetro do colmo $(\mathrm{cm})$ de plantas de milho (Zea mays L. - LG 6036), em função dos dias após a germinação $(15,30,45,60,75$, 90, 105 e 120 dias). Urutaí, GO, 2014.

\begin{tabular}{|c|c|c|c|c|c|c|}
\hline \multirow[b]{2}{*}{ Modelos de regressão } & \multicolumn{6}{|c|}{ Irrigação com água de abastecimento (A) } \\
\hline & $\begin{array}{c}\text { Solo } \\
\text { (testemunha) }\end{array}$ & $\begin{array}{l}\text { Solo + } \\
\text { NPK }\end{array}$ & $\begin{array}{l}\text { Solo + } \\
\text { VLp20 }\end{array}$ & $\begin{array}{c}\text { Solo + } \\
\text { VLp20 + P }\end{array}$ & $\begin{array}{l}\text { Solo + } \\
\text { VLc20 }\end{array}$ & $\begin{array}{c}\text { Solo + } \\
\text { VLc20 + P }\end{array}$ \\
\hline Reg. linear & 0,53 & 0,77 & 0,87 & 0,75 & 0,66 & 0,68 \\
\hline Reg. quadrática & 0,86 & 0,98 & 0,99 & 0,97 & 0,95 & 0,93 \\
\hline MLRP & - & - & - & - & - & - \\
\hline MQRP & - & 0,99 & 0,99 & 0,98 & 0,98 & 0,98 \\
\hline \multirow[t]{2}{*}{$\mathrm{CV}(\%)$} & 34,66 & 15,89 & 11,13 & 7,70 & 16,80 & 11,33 \\
\hline & \multicolumn{6}{|c|}{ Irrigação com água residuária (R) } \\
\hline Reg. linear & 0,84 & 0,71 & 0,68 & 0,65 & 0,66 & 0,52 \\
\hline Reg. quadrática & 0,98 & 0,95 & 0,96 & 0,95 & 0,96 & 0,90 \\
\hline MLRP & - & - & - & - & - & 0,98 \\
\hline MQRP & 0,98 & 0,99 & 0,99 & 0,99 & 0,99 & - \\
\hline $\mathrm{CV}(\%)$ & 8,34 & 7,21 & 9,02 & 12,68 & 5,06 & 11,63 \\
\hline
\end{tabular}

Legenda: (-): modelo não ajustado; Reg: regressão; MLRP: modelo de regressão linear de resposta platô; MQRP: modelo de regressão quadrática de resposta platô.

No entanto, o modelo de regressão que melhor se ajustou à maioria dos resultados referentes a esta variável foi o de regressão quadrática com resposta platô (Figura 2), com valores de $\mathrm{R}^{2}$ iguais ou superiores a $98 \%$.

Os dados do tratamento T1A (irrigado com água de abastecimento) seguiram o modelo de regressão linear simples e os do tratamento T6R (irrigado com água residuária) seguiram o modelo de regressão linear com resposta platô (Figura 2), apresentando comportamento semelhante ao observado para a variável altura da planta (Figura 1).

Os dados de altura e diâmetro do colmo das plantas obtidos nesta pesquisa corroboram outros estudos que, em conjunto ou isoladamente, avaliaram os efeitos da água residuária e do uso de vermicompostos no incremento da cultura de plantas de milho. Costa et al. (2009), ao quantificarem os efeitos isolados e consorciados da irrigação com água residuária e de doses de biossólidos no crescimento do milho (cultivar BR-106), após o cultivo da mamona, verificaram que todas as variáveis de crescimento das plantas de milho foram superiores para os tratamentos que receberam água residuária. Javarez-Junior et al. (2010) verificaram não apenas o melhor desenvolvimento da cultura do milho (Cultivar AL Bandeirante), irrigado com efluente de esgoto doméstico, como também a melhor produtividade. 
Quanto aos estudos que avaliaram o uso de vermicompostos no crescimento do milho, destacam-se os trabalhos de Chandrashekara et al. (2000), Brunes et al. (2008a; 2008b), Lazcano et al. (2011) e De e Bandyopadhyay (2013). Esses estudos, de modo geral, demonstraram que o acréscimo de vermicompostos, em quantidades adequadas e planejadas aos solos de cultivo, proporcionam efeitos positivos sobre o crescimento da cultura do milho. No que diz respeito ao uso de vermicompostos de lodo de curtume na cultura do milho, constatou-se grande lacuna na literatura especializada, fato este que dificulta enormemente a discussão dos dados obtidos no presente trabalho, pela não existência de outros resultados publicados com abordagem semelhante.

Encontrou-se apenas o trabalho de Gondek e Filipek-Mazur (2003), no qual os autores avaliaram o efeito de três diferentes vermicompostos de lodo de curtume, sobre o aumento da biomassa aérea e da raiz de plantas de milho (cultivar KLG 2210). O referido estudo apontou que no primeiro ano após a aplicação dos vermicompostos, o efeito dos compostos no aumento da biomassa das plantas de milho foi igual ao do tratamento que recebeu apenas esterco bovino, mas significativamente inferior em relação ao tratamento com adubação mineral.

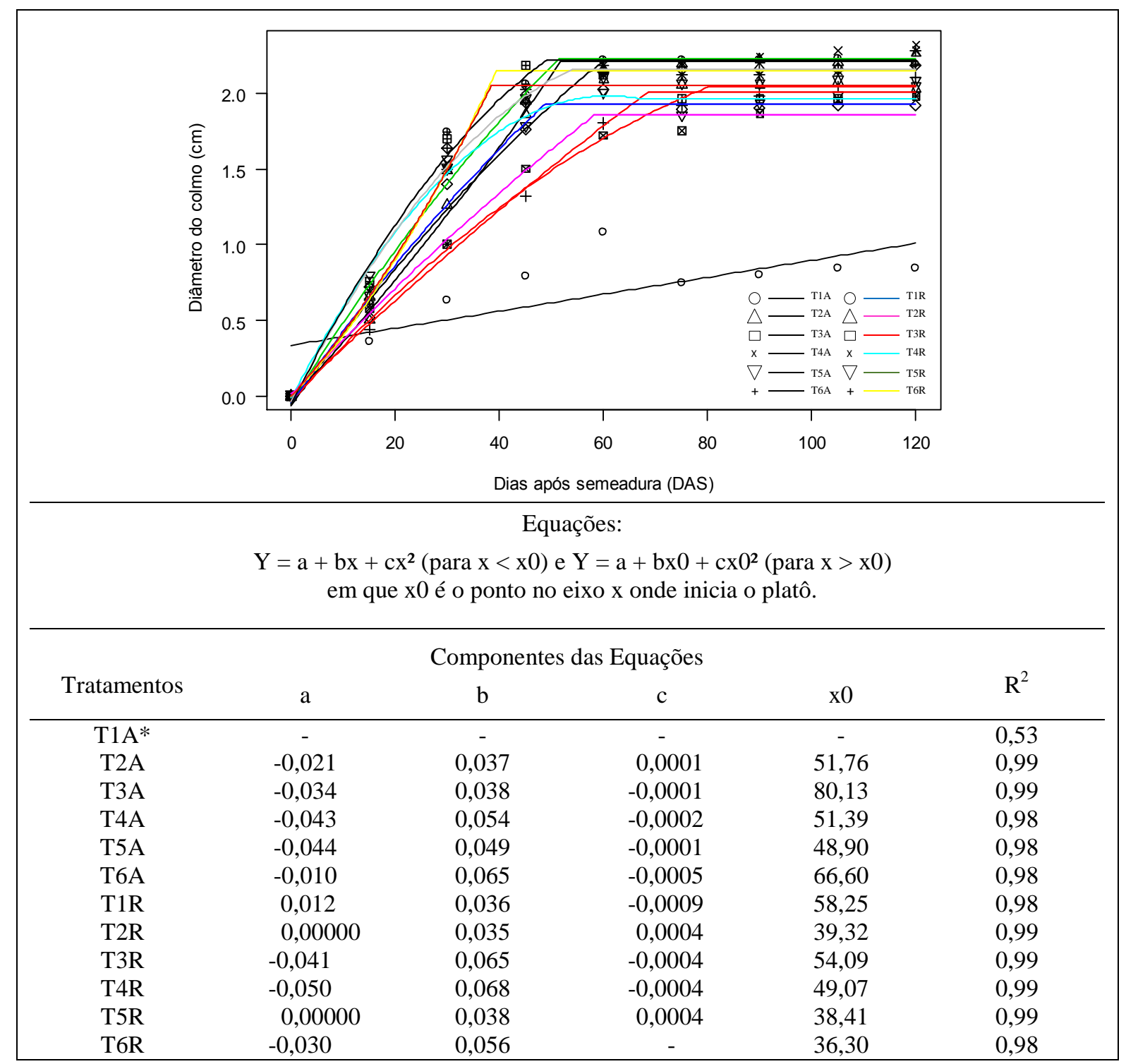

Figura 2. Representação gráfica e equações dos modelos de regressão que melhor se ajustaram aos resultados de diâmetro do colmo (cm) de plantas de milho (Zea mays - LG 6036), obtidos ao longo do período experimental. Urutaí, GO, 2014. Legenda: $\mathrm{R}^{2}$ : Coeficientes de determinação. 
A diferença dos resultados obtidos por Gondek e Filipek-Mazur (2003), em relação aos da presente pesquisa, pode estar relacionada, dentre outros fatores, à composição dos vermicompostos testados. Na presente pesquisa foram usados vermicompostos de lodo de curtume misturados a esterco bovino, numa proporção de $20 \%$ de lodo. No entanto, aqueles autores produziram três tipos de vermicompostos a partir de misturas de lodo de curtume com folhagem de coníferas, papelão e palha de trigo.

\section{CONCLUSÃO}

Com base nos resultados e de acordo com as condições experimentais, pode-se concluir que vermicompostos de lodo de curtume e irrigação com água residuária de origem doméstica no cultivo do milho proporcionaram maiores altura e diâmetro caulinar às plantas de milho, ao longo do experimento.

\section{AGRADECIMENTO}

Agradecemos o Instituto Federal Goiano, Câmpus Urutaí pelo apoio à pesquisa.

\section{REFERÊNCIAS}

AGÊNCIA NACIONAL DAS ÁGUAS (Brasil). Evolução dos recursos hídricos no Brasil: regiões hidrográficas do Brasil, caracterização geral e aspectos prioritários, relatório de gestão 2001. Brasília, 2002. 72 p.

ANDRADE-FILHO, J. A.; SOUSA, O. N.; DIAS, N. S.; NASCIMENTYO, I. B.; MEDEIROS, J. F.; COSME, C. R. Atributos químicos de solo fertirrigado com água residuária no semiárido brasileiro. Irriga, Botucatu, v. 18, n. 4, p. 661-674, out./dez. 2013. http://dx.doi.org/10.15809/irriga.2013v18n4p661

AMERICAN PUBLIC HEALTH ASSOCIATION - APHA; AMERICAN WATER WORKS ASSOCIATION - AWWA; WATER ENVITONMENT FEDERATION - WEF. Standard methods for the examination of water and wastewater. 20. ed. New York, 1997. $1194 \mathrm{p}$.

AQUINO, A. M.; ALMEIDA, D. L.; SILVA, V. F. Utilização de minhocas na estabilização de resíduos orgânicos: vermicompostagem. Rio de Janeiro: Embrapa Embrapa/CNPBS. 1992. 12 p.

BONINI, M. A.; SATO, L. M.; BASTOS, R. G.; SOUZA, C. F. Alterações nos atributos químicos e físicos de um Latossolo Vermelho irrigado com água residuária e vinhaça. Revista Biociências, Taubaté, v. 20, n. 1, p. 56-63, jan. 2014.

BRUNES, A. P.; CASTILHOS, D. D.; CASTILHOS, R. M. V.; MORAES, J. R.; SANTOS, K. F. Crescimento de milho e alterações químicas do solo após a aplicação de vermicompostos. In: REUNIÃO BRASILEIRA DE FERTILIDADE DO SOLO E NUTRIÇÃO DE PLANTAS, REUNIÃO BRASILEIRA SOBRE MICORRIZAS; SIMPÓSIO BRASILEIRO DE MICROBIOLOGIA DO SOLO; REUNIÃO BRASILEIRA DE BIOLOGIA DO SOLO, 2008, Londrina. Anais... Londrina, PR: SBCS, 2008a.

BRUNES, A. P.; CASTILHOS, D. D.; CASTILHOS, R. M. V.; MORAES, J. R.; SANTOS, K. F. Estado nutricional de plantas de milho cultivadas em solo acrescido de vermicomposto. In: CONGRESSO DE INICIAÇÃO CIENTÍFICA, X ENCONTRO DE PÓS-GRADUAÇÃO, 17., 2008, Pelotas. Anais.... Pelotas: UFPel, 2008 b. 
CHANDRASHEKARA, S. I.; HARLAPUR, S.; MURALIKRISHAN, M.; GIRIJESHM, G. $\mathrm{K}$. Response of maize to organic manures with inorganic fertilizers. Karnataka Journal of Agricultural Sciences, Karnataka, v. 13, n. 5, p. 144-146, Sept. 2000.

COSTA, F. X.; LIMA, V. L. A.; BELTRÃO, N. E. M.; AZEVEDO, C. A. V.; SOARES, F. A.; ALVA, I. D. M. Efeitos residuais da aplicação de biossólidos e da irrigação com água residuária no crescimento do milho. Revista Brasileira de Engenharia Agrícola e Ambiental, Campina Grande, v. 13, n. 6, p. 687-693, nov./dez. 2009. http://dx.doi.org/10.1590/S1415-43662009000600004

DE, B.; BANDYOPADHYAY, S. Influence of soil conservation techniques on growth and yield of maize (Zea mays L.) in Terai region of West Bengal. SAARC Journal of Agriculture, Dhaka, v. 11, n. 1, p. 133-147, jan. 2013.

http://dx.doi.org/10.3329/sja.v11i1.18390

EMPRESA BRASILEIRA DE PESQUISA AGROPECUÁRIA. Centro Nacional de Pesquisa de Solos. Manual de métodos de análise de solo. 2. ed. Brasília, 1997. 212 p.

FONSECA, A. F.; MELFI, A. J.; MONTES, C. R. Maize growth and changes in soil fertility after irrigation with treated sewage effluent. I. Plant dry matter yield and soil nitrogen and phosphorus availability. Communications in Soil Science and Plant Analysis, v. 36, n. 13-14, p. 1965-1981, fev. 2005a. http://dx.doi.org/10.1081/CSS-200062539

FONSECA, A. F.; MELFI, A. J.; MONTES, C. R. Maize growth and changes in soil fertility after irrigation with treated sewage effluent. II. Soil acidity, exchangeable cations, and sulfur, boron, and heavy metals availability. Communications in Soil Science and Plant Analysis, v. 36, n. 13-14, p. 1983-2003, fev. 2005 b.

http://dx.doi.org/10.1081/CSS-200062542

FONSECA, A. F.; LEAL, R. M. P.; HERPIN, U.; MELFI, A. J. Carbon and nitrogen dynamics in a Brazilian soil-pasture system irrigated with treated sewage effluent. Israel Journal of Plant Sciences, Jerusalem, v. 59, n. 2-4, p. 147-157, mar. 2011. http://dx.doi.org/10.1560/IJPS.59.2-4.147

GANEM, R. S. Curtumes: aspectos ambientais. 2007. Disponível em: http://bd.camara.gov.br/bd/bitstream/handle/bdcamara/1281/

curtumes_aspectos_senna.pdf?sequence=1. Acesso em: 20 maio 2013.

GODECKE, M. V.; RODRIGUES, M. A. S.; NAIME, R. H. Resíduos de curtume: estudo das tendências de pesquisa. Revista Eletrônica em Gestão, Educação e Tecnologia Ambiental, Santa Maria, v. 7, n. 7, p. 1357-1378, mar./ago. 2012. http://dx.doi.org/10.5902/22361170

GONDEK, K.; FILIPEK-MAZUR, B. Biomass yields of shoots and roots of plants cultivated in soil amended by vermicomposts based on tannery sludge and content of heavy metals in plant tissues. Plant Soil and Environment, v. 49, n. 9, p. 402-409, nov. 2003.

HESPANHOL, I. Potencial uso de água no Brasil: agricultura, indústria, municípios, recarga de aquíferos. Revista Bahia Análise e Dados, Salvador, v. 13, n. Especial, p. 411-437, dez. 2003.

JAVAREZ-JUNIOR, A.; RIBEIRO, T. A. P; PAULA-JUNIOR, D. R. Eficiência do reuso de águas residuárias na irrigação da cultura do milho. Irriga, Botucatu, v. 15, n. 3, p. 231247, jul./set., 2010. http://dx.doi.org/10.15809/irriga.2010v15n3p231 
KRAEMER, M. E. P. A questão ambiental e os resíduos industriais. Disponível em: <http://www.amda.org.br/imgs/up/Artigo_25.pdf.> Acesso em: 14 nov. 2014.

LAZCANO, C.; REVILLA, P.; MALVAR, R. A.; DOMÍNGUEZ, J. Yield and fruit quality of four sweet corn hybrids (Zea mays) under conventional and integrated fertilization with vermicompost. Journal of the Science of Food and Agriculture, New Zealand, v. 91, n. 7, p. 1244-1253, feb. 2011. http://dx.doi.org/10.1002/jsfa.4306

LEAL, R. M. P.; FONSECA, A. F.; HERPIN, U.; MELFI, A. J. Agricultural utilization of treated sewage effluent: experience from Brazil. Israel Journal of Plant Science, Jerusalem, v. 59, n. 2-4, p. 235-248, jun. 2011. http://dx.doi.org/10.1560/IJPS.59.2.xx

LUERSEN, L. H.; SHULTZ, G.; REMPEL, C. Reuso do efluente tratado na indústria curtumeira. Tecno-Lógica, Santa Cruz do Sul, v. 16, n. 1, p. 5-10, jan./jun. 2012.

MONTEIRO, D. R.; SILVA, T. T. S.; SILVA, L. V. B. D.; LIMA, V. L. A.; SANTOS, C. L. M.; PEARSON, H. W. Efeito da aplicação de efluente doméstico tratado nos teores de micronutrientes no solo. Irriga, Botucatu, v. Especial, n. Especial, p. 40-46, dez. 2014. http://dx.doi.org/10.15809/irriga.2014v1n1p40

NUNES, P. M. P.; SMOLAREK, F. S. F.; KAMINSKI, G. A. T.; FIN, M. T.; ZANIN, S. M. W.; MIGUEL, M. D. et al. A importância do aproveitamento dos resíduos industriais da semente de Citrus. Visão Acadêmica, Curitiba, v. 10, n. 1, p. 97-110, jan/jun. 2009. http://dx.doi.org/10.5380/acd.v10i1.21324

PACHECO, J. W. F. Curtumes: Série P+L. 2005. Disponível em: http://www.cetesb.sp.gov.br Acesso em: 5 jan. 2013

PACHECO, J. W. F. Curtumes. São Paulo: CETESB, 2009.

PINHEIRO, F. G. R. Avaliação do potencial poluidor de curtumes do Distrito Industrial de Icoaraci e influência sobre os recursos hídricos locais. 2005. 140f. Dissertação (Mestrado em Geologia) - Centro de Geociências. Universidade Federal do Pará, Belém, 2005.

R CORE TEAM. R: a language and environment for statistical computing. Vienna: $\mathrm{R}$ Foundation for Statistical Computing. Disponível em: https://www.r-project.org/ Acesso em: 6 mar. 2014.

SALOMÃO, L. C. Calibração de tanques evaporímetros de baixo custo sob diferentes diâmetros em ambiente protegido. 2012. 74f. Tese (Doutorado em Agronomia) Faculdade de Ciências Agronômicas da Universidade Estadual Paulista "Júlio de Mesquita Filho", Botucatu, 2012.

SILVA, L. L.; CARVALHO, C. M.; SOUZA, R. P. F.; FEITOSA, H. O.; FEITOSA, S. O.; GOMES FILHO, R. R. Utilização de efluentes domésticos no crescimento da pimenta (Capsicum chinense), cultivar tequila bode vermelha. Revista AGROTEC, Porto, v. 35, n. 1, p. 121-133, jan./fev. 2014.

SOUSA, D. M. G.; LOBATO, E. Calagem e adubação para culturas anuais e semiperenes. In: SOUSA, D. M. G.; LOBATO E. (Eds.). Cerrado: correção do solo e adubação. 2. ed. Planaltina: Embrapa Cerrado, 2004. p. 283-315.

TEDESCO, M. J.; GIANELlO, C.; BISSANI, C.; BOHNEN, H.; VOLKWEISS, S. J. Análise de solo, plantas e outros materiais. 2. ed. Porto Alegre: Universidade Federal do Rio Grande do Sul, 1995. 174 p. 
VIG, A. P.; SINGH, J.; WANI, S. H.; DHALIWAL, S. S. Vermicomposting of tannery sludge mixed with cattle dung into valuable manure using earthworm Eisenia fetida (Savigny). Bioresource Technology, v. 102, n. 17, p. 7941-7945, set. 2011.

http://dx.doi.org/10.1016/j.biortech.2011.05.056 